\title{
Efectos proinflamatorios de la contaminación atmosférica
}

\author{
MANUEL OYARZÚN G.*, NELSON DUSSAUBAT D.*, M. EUGENIA MILLER A.* \\ SILVIA LABRA J.** y SERGIO GONZÁLEZ B.***
}

\section{Proinflammatory effects of air pollution in Santiago de Chile}

Intermittent exposure of rats to Santiago's traffic pollution is associated to a decrease in growth after more than 100 days (range: 101-111) and to histological lung damage after 90 and particularly after 180 days. Our aim was to assess whether a 90 days exposure of rats to air from a Santiago's heavy traffic avenue, is able to induce a systemic proinflammatory reaction. Thirty-days-old Sprague-Dawley rats $(n=7)$ were directly exposed to air from a heavy traffic avenue ( 8 h, 5 days a week, from April 27 to July 29, 2009). Controls $(n=7)$ breathed animal room air. Rats were weighed twice a week and after completing 90 days of observation, lungs were subjected to histopathology and $C$ reactive protein, viscosity and $F_{2}$-isoprostane in plasma and microhematocrit were determined in blood samples. Exposure to $\mathrm{PM}_{10}, \mathrm{PM}_{2.5}$, ozone, $\mathrm{NO}_{2}$ and $\mathrm{CO}$ were estimated from registrations of 4 Santiago's monitoring stations. Plasmatic $C$ reactive protein and viscosity and microhematocrit were significantly increased after 90 days of exposure as compared to controls $(p<0.05)$. No significant changes were observed in $F_{2}$-isoprostane, nor in lung histopathology, nor in body weight curve versus time in exposed as compared to control series. Hourly mean value of PM 2.5 in the 8 h of exposure was high: $38.9 \mu \mathrm{g} / \mathrm{m}^{3}$. It is concluded that 90 days of intermittent exposure of rats to Santiago's air pollution would promote a systemic inflammatory reaction. This response to air pollution might precede the decrease in body growth and the histological lung damage reported previously by our laboratory in the same species after intermittent Santiago's urban air pollution exposure.

Key words: Air pollution, systemic inflammatory reaction, intermittent exposure, sentinel rats.

\section{Resumen}

La exposición intermitente de ratas centinela a la contaminación del tráfico vehicular de Santiago se ha asociado a disminución del crecimiento corporal después de cien días de exposición (rango: 101-111) y a daño histopatológico del pulmón a los 90 días y más, especialmente a los 180 días de exposición. Nuestro objetivo fue evaluar si la exposición al aire de una avenida con elevado tráfico vehicular durante 90 días era capaz de inducir en la rata una respuesta inflamatoria sistémica. Ratas Sprague-Dawley de 30 días de edad $(n=7)$ fueron directamente expuestas a respirar el aire de una avenida con elevado flujo vehicular (8 h, 5 días por semana, desde el 27 de abril hasta el 29 de julio de 2009). Las ratas control $(n=7)$ respiraron aire del bioterio. Las ratas se pesaron dos veces por semana y después de completar 90 días de observación, los pulmones se destinaron a estudio histopatológico. Se realizó microhematocrito y se determinó proteína $C$ reactiva, viscosidad y $F_{2}$-isoprostano plasmáticos en muestras de sangre. La exposición a PM $\mathrm{PM}_{10}, \mathrm{PM}_{2,5}$, ozono, $\mathrm{NO}_{2}$ y $\mathrm{CO}$ se calculó de los registros de cuatro estaciones de monitoreo de Santiago. Después de 90 días de exposición se observó un aumento significativo $(p<0,05)$ de la proteína $C$ reactiva y de la viscosidad plasmática y también del microhematocrito, en relación a la serie control. No se observaron cambios significativos en $F_{2^{-}}$ isoprostano plasmático, ni en la histopatología pulmonar, ni en la curva de peso corporal versus tiempo al comparar la serie expuesta con la serie control. El promedio horario de $P M_{2,5}$ en las 8 horas de

\footnotetext{
* Programa de Fisiopatología, ICBM, Facultad de Medicina, Universidad de Chile ** Laboratorio de Hematología, Hospital del Salvador.

*** Departamento de Anatomía Patológica. Pontificia Universidad Católica de Chile.
} 
exposición fue alto: $38,9 \mu \mathrm{g} / \mathrm{m}^{3}$. Concluimos que 90 días de exposición intermitente a la contaminación aérea de Santiago en el modelo experimental promueve una reacción inflamatoria sistémica. Esta respuesta a la contaminación aérea podría preceder a la disminución del crecimiento corporal y al daño histológico pulmonar encontrado en otro de nuestros estudios en esta misma especie después de la exposición intermitente a la contaminación aérea de Santiago.

Palabras clave: Contaminación atmosférica, reacción inflamatoria sistémica, exposición intermitente, ratas centinela.

\section{Introducción}

El tráfico vehicular es una de las principales fuentes de contaminación atmosférica en Santiago, contribuyendo importantemente en la producción de material particulado $\left(\mathrm{PM}_{10}\right.$ y $\left.\mathrm{PM}_{2,5}\right)$, como también de monóxido de carbono (CO), de dióxido de nitrógeno $\left(\mathrm{NO}_{2}\right)$ y de compuestos orgánicos volátiles $(\mathrm{COV})$ que son precursores de ozono que es un contaminante secundario, generado por el efecto de la radiación solar sobre los $\mathrm{COV}$ y $\mathrm{NO}_{2}{ }^{1}$.

El inventario de emisiones de contaminantes atmosféricos realizado en Santiago en el año 2000, demostró que las fuentes móviles contribuyen con el $68,2 \%$ del total de las emisiones a la atmósfera, constituyéndose en uno de los aportes más significativos y más susceptibles de variación, producto de la evolución que tiene el sector transporte ${ }^{1}$.

En un estudio previo ${ }^{2}$ encontramos que la exposición intermitente de ratas centinelas a contaminantes atmosféricos derivados principalmente del tráfico vehicular urbano, se asoció a daño pulmonar detectable por aumento de células y proteínas en el lavado broncoalveolar a los 90 días de exposición y por la presencia de macrófagos peribronquiales en el estudio histopatológico luego de 180 días de exposición. También se observó disminución de peso corporal después de cien días de exposición (rango: 101-111 días), esta disminución del peso corporal, representa una disminución significativa de la curva de crecimiento de la serie expuesta, lo cual sugiere que la inhalación de aire urbano de Santiago no sólo afectaría al pulmón, su puerta de entrada, sino que también al resto del organismo. En este sentido la baja de peso revelaría un compromiso del estado general que podría estar relacionado con el aumento de las pérdidas insensibles de agua (aumento de la ventilación minuto) y eventualmente del metabolismo (estado proinflamatorio). Pope y colaboradores han sugerido que el riesgo de mortalidad cardiopulmonar por exposición a material particulado del aire estaría asociado a una reacción inflamatoria pulmonar y sistémica ${ }^{3}$. Por otra parte, en seres humanos en episodios de contaminación atmosférica por material particulado se ha detectado aumento de la viscosidad plasmática ${ }^{4} \mathrm{y}$ de la concentración plasmática de la proteína $\mathrm{C}$ reactiva, que habitualmente aumenta en la fase aguda de la inflamación ${ }^{5}$.

En este contexto el propósito de este estudio fue conocer si en estas ratas centinela de la contaminación atmosférica urbana se producía un efecto proinflamatorio sistémico luego de 90 días de exposición intermitente al aire de una avenida con intenso tráfico vehicular.

El uso de animales como centinelas de los riesgos ambientales para la salud humana ha sido discutido en otras publicaciones. Se ha sugerido que a pesar que es improbable que los datos obtenidos en diferentes especies sean usados como factores determinantes únicos en la evaluación de efectos de contaminantes ambientales sobre la salud humana, tales datos pueden ser útiles para aumentar el peso de la evidencia en la evaluación de riesgos. Estos estudios también pueden servir para generar preocupación sobre situaciones que requieren estudios complementarios o para monitorizar el curso de actividades o medidas que tienen por objetivo remediar la contaminación ambiental y sus efectos ${ }^{6}$.

\section{Material y Métodos}

Se utilizaron 14 ratas macho Sprague-Dawley de 30 días de edad y de peso promedio inicial $105,6 \mathrm{~g} \pm 5,2 \mathrm{DS}$. Las ratas recibieron permanentemente alimento adecuado para sus requerimientos metabólicos (Champion S.A.) y suministro de agua ad libitum. Las ratas fueron separadas en dos grupos: expuesto $(\mathrm{n}=7$; peso promedio inicial: $105,7 \pm 5,4 \mathrm{~g})$ y control $(\mathrm{n}=7$; peso promedio inicial: $105,4 \pm 5,38 \mathrm{~g}$ ), ambos grupos fueron observados durante 90 días.

Las ratas fueron expuestas durante 90 días (desde el 27 de abril hasta el 29 de julio de 2009), ocho horas diarias (de 09:00 a 17:00 h), 5 días por semana (lunes a viernes) al aire de Avda. Salvador, Comuna de Providencia. Para lo cual fueron ubicadas en sus jaulas en un espacio especialmente adaptado para este propósito, en 
el marco de una ventana del edificio (Avda. Salvador 486), ubicado a $1,5 \mathrm{~m}$ del suelo y a $5 \mathrm{~m}$ de la calzada que tiene un elevado tráfico vehicular (1.700 vehículos por hora entre las 09:00 y 10:00 h). La temperatura de cada jaula de exposición se mantuvo entre 20 y $22{ }^{\circ} \mathrm{C}$ mediante el uso de lámparas de luz infrarroja. Al término de las 8 horas diarias de exposición, las ratas se trasladaban en su jaula al cubículo del bioterio en el que estaba el grupo control, con temperatura controlada entre 20 y $22{ }^{\circ} \mathrm{C}$ y con ciclo luz/oscuridad de 12 horas. Las ratas fueron pesadas dos veces por semana para comparar la curva de crecimiento de ambos grupos.

Al término de los noventa días de observación las ratas controles y expuestas fueron sedadas con Acepromazina $(0,4 \mathrm{mg} / \mathrm{kg}$ de peso i.m.) y anestesiadas con Ketamina (20 mg/kg i.m.) asociada a Atropina $(0,04 \mathrm{mg} / \mathrm{kg}$ i.m.) para evitar el broncoespasmo y una vez que se logró un nivel anestésico adecuado fueron eutanasiadas mediante exanguinación por punción de la aorta abdominal.

La sangre extraída fue conservada en un tubo de ensayo con anticoagulante (EDTA o heparina según la determinación a efectuar) para realizar microhematocrito, medición de viscosidad plasmática, determinación de $\mathrm{F}_{2}$-isoprostano $\mathrm{y}$ de proteína $\mathrm{C}$ reactiva.

La viscosidad plasmática se midió por el tiempo (segundos) que demora el plasma en recorrer una distancia predeterminada $(7,8 \mathrm{~cm})$ en el capilar de una pipeta de radio conocido en condiciones controladas de presión atmosférica, posición $\left(13,3^{\circ}\right.$ de inclinación sobre el plano horizontal) y temperatura entre 20 y $25^{\circ} \mathrm{C}$. Este método había sido previamente estandarizado en el Laboratorio de Hematología del Hospital del Salvador, usando muestras plasmáticas de viscosidad conocida.

La concentración plasmática de $\mathrm{F}_{2}$-isoprostano se realizó por enzimoinmunoensayo (ELISA) usando un kit de Cayman Chemicals Co., Michi$\operatorname{gan}^{7}$. La determinación de proteína $\mathrm{C}$ reactiva se efectuó en muestras de plasma mediante una prueba fototurbidimétrica ${ }^{8}$.

Los pulmones fueron extraídos del tórax mediante una esternotomía media para efectuar en ellos un estudio histopatológico, a cargo del patólogo del equipo (SGB) quien evaluó el pulmón desconociendo la serie a que pertenecía cada muestra. Las muestras fueron fijadas con formalina al 10\% tamponada a $\mathrm{pH} 7$ y luego teñidas con hematoxilina-eosina.

El manejo de las ratas se llevó a cabo ateniéndose estrictamente a las guías para el uso y cuidado de animales de la American Physiological Society. El protocolo de este estudio fue aprobado por el Comité de Bioética sobre investigación en animales de la Facultad de Medicina, Universidad de Chile (Protocolo CBA \#0307).

En el análisis estadístico de los resultados se utilizó t-test con o sin corrección de Welch según el caso. Un valor de $\mathrm{p} \leq 0,05$ fue considerado significativo.

\section{Medición de la exposición}

Los niveles de contaminantes atmosféricos durante el período de exposición fueron calculados de los valores obtenidos en cuatro estaciones de la red de monitoreo de contaminantes atmosféricos de la Región Metropolitana (Las Condes, La Paz, Parque O'Higgins y La Florida). Estos valores fueron proporcionados por el Centro Nacional del Medio Ambiente (CENMA) de la Universidad de Chile. Para el cálculo de cada contaminante ( $\mathrm{PM}_{10}, \mathrm{PM}_{2.5}$, ozono, $\mathrm{NO}_{2}$ y $\left.\mathrm{CO}\right) \mathrm{se}$ aplicó la siguiente ecuación:

$$
\mathrm{x}=\frac{\Sigma(\mathrm{c} \cdot \mathrm{w})}{\Sigma \mathrm{w}}
$$

Siendo " $\mathrm{x}$ " la concentración estimada del contaminante en el lugar de exposición; "c" la concentración medida en cada una de las 4 estaciones monitoras; $\mathrm{w}=1 / \mathrm{d}$ siendo " $\mathrm{d}$ " la distancia $(\mathrm{km})$ entre la estación monitora y la zona de exposición.

\section{Resultados}

Al comparar las concentraciones de contaminantes estimadas en el sitio de medición con su respectiva norma nacional ${ }^{9}$, se puede observar que la concentración de $\mathrm{PM}_{10}$ sistemáticamente excedió la norma anual $\left(50 \mu \mathrm{g} / \mathrm{m}^{3}\right)$ y frecuentemente superó la norma diaria $\left(150 \mu \mathrm{g} / \mathrm{m}^{3}\right)$ (Figura 1). La concentración horaria promedio de $\mathrm{PM}_{10}$ en el período de 8 horas de exposición fue de $83,1 \mu \mathrm{g} / \mathrm{m}^{3}$. Por su parte la concentración horaria promedio de $\mathrm{PM}_{2,5}$ en el período de exposición fue de $38,9 \mu \mathrm{g} / \mathrm{m}^{3}$ excediendo frecuentemente (Figura 2) la norma anual $\left(25 \mu \mathrm{g} / \mathrm{m}^{3}\right)$ propuesta por la Comisión Nacional del Medio Ambiente $(\text { CONAMA })^{10}$.

Durante el período de exposición, las concentraciones de $\mathrm{NO}_{2}$ alcanzaron un valor promedio por hora de $37,7 \mu \mathrm{g} / \mathrm{m}^{3}$ y sólo en una oportunidad superó el valor de la norma anual de $100 \mu \mathrm{g} / \mathrm{m}^{3}$ (Figura 3). El CO siempre estuvo por debajo de $10 \mathrm{mg} / \mathrm{m}^{3}$ que es la norma nacional para ocho horas de exposición; durante el período de exposición su concentración promedio por hora fluctuó 

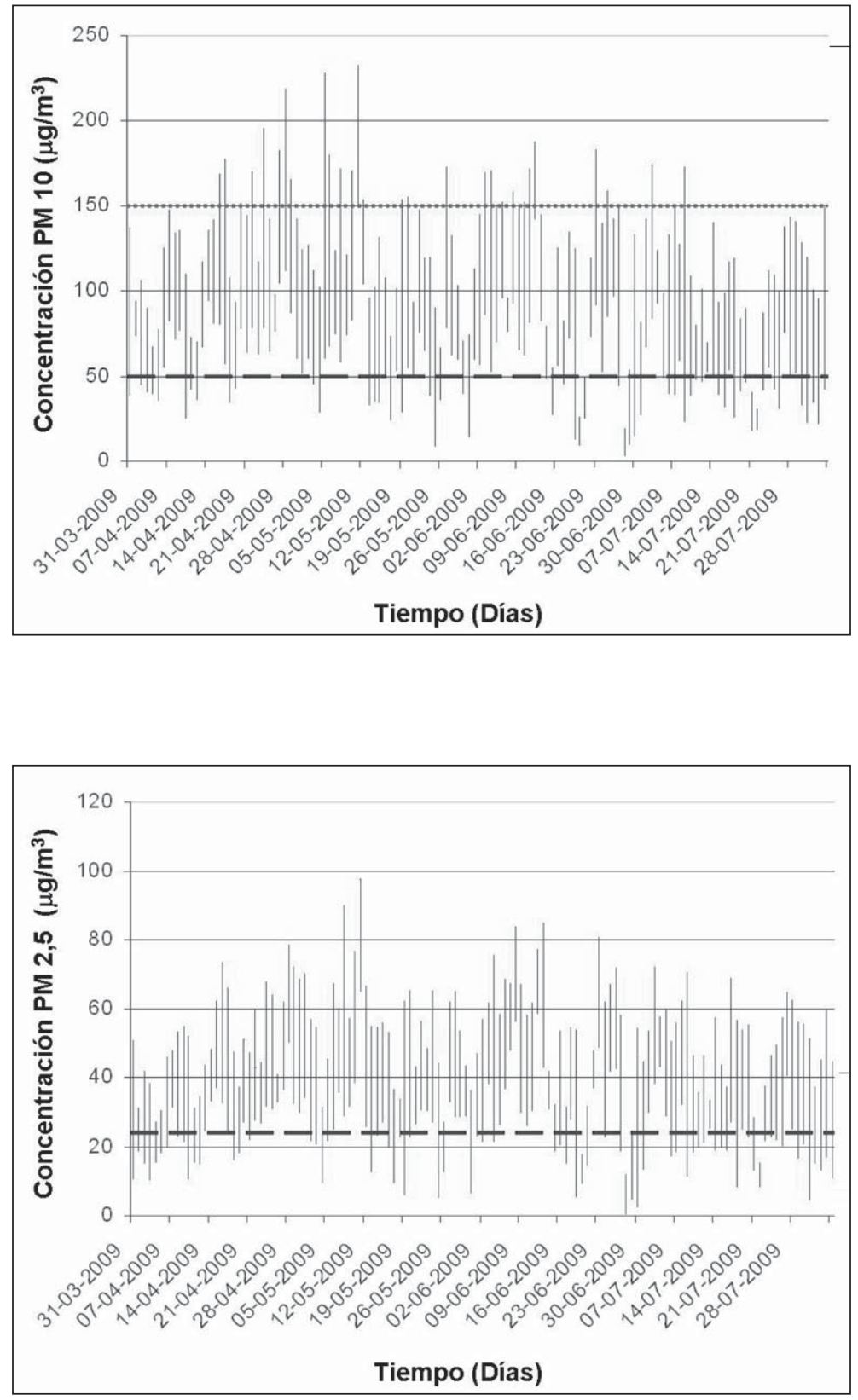

Figura 1. Concentraciones de $\mathrm{PM}_{10}$ entre abril y julio de 2009 calculadas para el sitio de exposición. Cada línea vertical representa dos valores de concentración obtenidos en un período de $24 \mathrm{~h}$ : el extremo superior de la línea corresponde a la concentración promedio por hora entre las 09:00 y las 17:00 h y el extremo inferior corresponde a la concentración promedio por hora entre las 18:00 y 08:00 h. La línea horizontal superior $(\cdots \cdots)$ representa la norma nacional diaria para $\mathrm{PM}_{10}\left(150 \mu \mathrm{g} / \mathrm{m}^{3}\right)$. La línea horizontal inferior (-----) representa la norma nacional anual para este contaminante $\left(50 \mu \mathrm{g} / \mathrm{m}^{3}\right)$.
Figura 2. Concentraciones de $\mathrm{PM}_{2,5}$ entre abril y julio de 2009 calculadas para el sitio de exposición. Cada línea vertical representa dos valores de concentración obtenidos en un período de $24 \mathrm{~h}$ : el extremo superior de la línea corresponde a la concentración promedio por hora entre las 09:00 y las 17:00 h y el extremo inferior corresponde a la concentración promedio por hora entre las 18:00 y 08:00 h. La línea horizontal (----) representa la norma anual propuesta por la CONAMA (10) para este contaminante $\left(25 \mu \mathrm{g} / \mathrm{m}^{3}\right)$.

Tabla 1. Microhematocrito, proteína $C$ reactiva, viscosidad y $F_{2}$-isoprostano plasmáticos en ratas controles y expuestas durante 90 días a la contaminación atmosférica de Providencia, Santiago

\begin{tabular}{|llcccc|}
\hline Series & & $\begin{array}{c}\text { Microhematocrito } \\
(\mathbf{\%})\end{array}$ & PCR (mg/L) & $\begin{array}{c}\text { Viscosidad } \\
\text { (s) }\end{array}$ & $\begin{array}{c}\text { F }_{2} \text {-isoprostano } \\
\text { (pg/L) }\end{array}$ \\
\hline Expuestas n $=7$ & Promedio & 39 & 17,2 & 7,7 & 56,2 \\
& DS & 0,58 & 1,7 & 0,49 & 23,7 \\
Controles n $=7$ & Promedio & 37 & 14,7 & 6,6 & 53,8 \\
& DS & 1,49 & 2,3 & 0,38 & 16,9 \\
\hline $\mathrm{p}^{*}$ & & 0,03 & 0,0438 & $<0,001$ & 0,836 \\
\hline
\end{tabular}

*p calculado con t-test de Student para muestras no pareadas. 

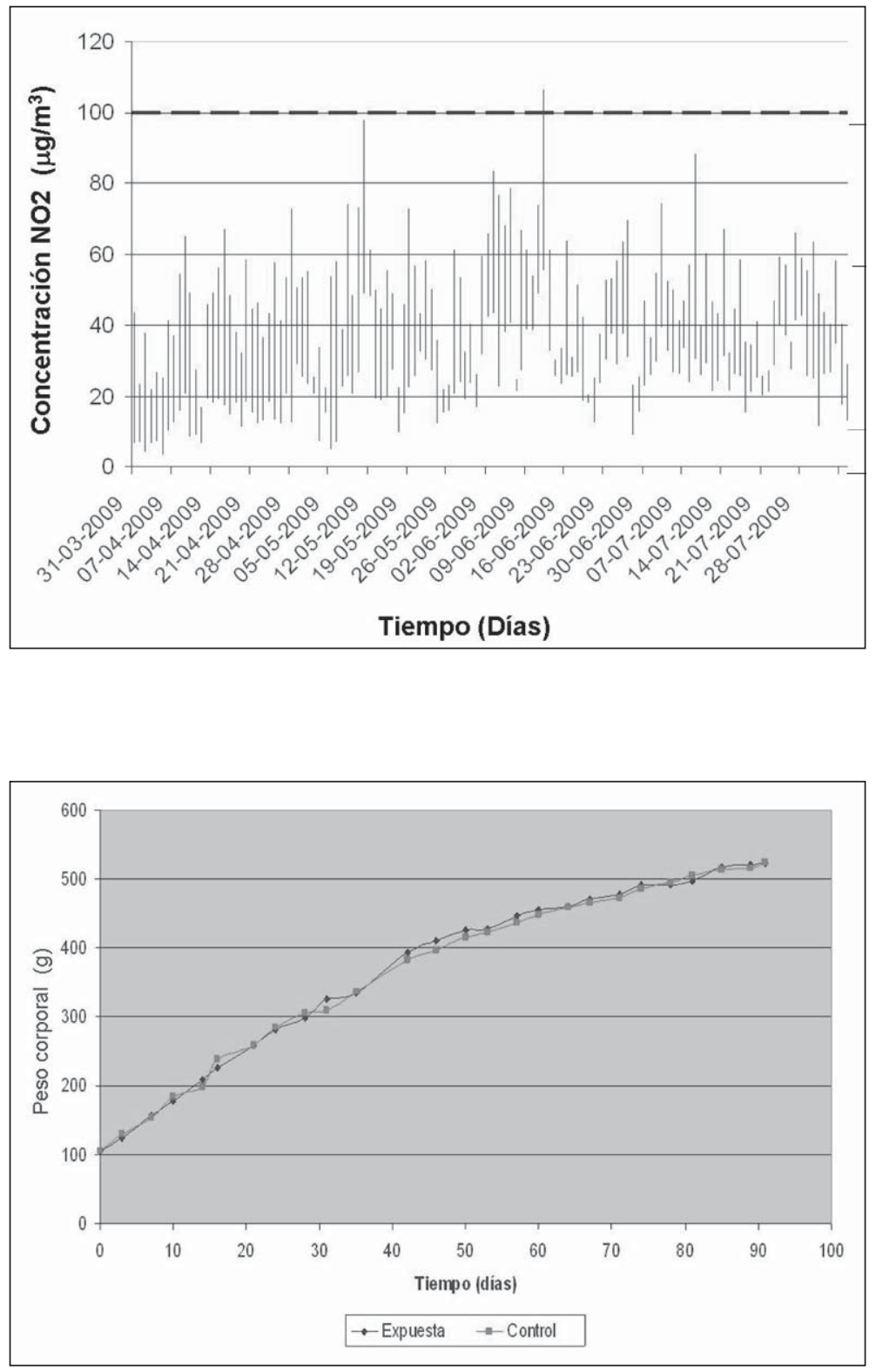

Figura 3. Concentraciones de $\mathrm{NO}_{2}$ entre abril y julio de 2009 calculadas para el sitio de exposición. Cada línea vertical representa dos valores de concentración obtenidos en un período de $24 \mathrm{~h}$ : el extremo superior de la línea corresponde a la concentración promedio por hora entre las 09:00 y las 17:00 h y el extremo inferior corresponde a la concentración promedio por hora entre las 18:00 y 08:00 h. La línea horizontal (-----) representa la norma nacional anual para este contaminante $\left(100 \mu \mathrm{g} / \mathrm{m}^{3}\right)$.
Figura 4. Curva de peso corporal durante los 90 días de observación en ratas de las series control y expuesta. Los puntos representan el valor promedio de cada curva. Las diferencias de peso corporal entre ambas curvas no fueron significativas ("t" test de Student para muestras no pareadas). entre 0,3 y $4 \mathrm{mg} / \mathrm{m}^{3}$. La concentración promedio de ozono en las $8 \mathrm{~h}$ de exposición fue de 10,9 $\mu \mathrm{g} /$ $\mathrm{m}^{3}$ por hora, fluctuando en un rango entre $1,4 \mathrm{y}$ $32,7 \mu \mathrm{g} / \mathrm{m}^{3}$, bastante alejado de la norma nacional para ozono $\left(120 \mu \mathrm{g} / \mathrm{m}^{3}\right.$ en $\left.8 \mathrm{~h}\right)$.

En comparación con sus controles, las ratas expuestas durante noventa días a la contaminación del tráfico urbano presentaron aumento significativo en la viscosidad plasmática $(\mathrm{p}=0,005)$, microhematocrito $(\mathrm{p}=0,03)$ y proteína $\mathrm{C}$ reactiva $(\mathrm{p}=0,04)$ (Tabla 1$)$.

La concentración plasmática de $\mathrm{F}_{2}$-isoprostano, un marcador biológico de estrés oxidativo ${ }^{11}$, no presentó una diferencia significativa entre las ratas expuestas y controles $(\mathrm{p}=0,84)$ (Tabla 1$)$ al igual que la curva de peso en que no se encontraron diferencias significativas durante todo el período (Figura 4). El estudio histopatológico pulmonar no demostró lesiones en las ratas controles ni expuestas.

\section{Discusión}

El propósito de este estudio fue evaluar el efecto proinflamatorio y el daño pulmonar en ratas centinelas expuestas a los contaminantes 
del tráfico vehicular en la zona del Hospital del Salvador, Santiago. Para lo cual se comparó ratas controles (nacidas y criadas en bioterio) con un grupo de estas mismas ratas que fueron expuestas intermitentemente y durante 90 días a la contaminación del aire de avenida Salvador, que tiene un intenso tráfico vehicular.

La curva de peso de las ratas expuestas fue similar a la de la serie control, lo cual está en concordancia con resultados de exposiciones previas de hasta 180 días, realizadas entre abril y septiembre de 2005 y 2007, en los que no se presentaron cambios significativos en los pesos de las ratas expuestas en relación con las controles antes de 100 días de exposición ${ }^{2}$.

La detección de daño pulmonar depende de la dosis efectiva, que a su vez es producto de la concentración de contaminantes, el tiempo de exposición y la ventilación minuto. En un estudio previo en ratas centinelas ${ }^{2}$ a los 90 días de exposición se detectó aumento de proteínas y de células totales en el lavado broncoalveolar acompañados de escasas y leves lesiones histopatológicas, las cuales se hicieron claramente evidentes al completar 180 días de exposición intermitente. En el presente estudio aunque no se efectuó lavado broncoalveolar, el estudio histopatológico del pulmón no detectó lesiones al cabo de 90 días de exposición. La ausencia de lesiones histopatológicas si bien no implica necesariamente ausencia de daño pulmonar, indicaría que el daño pulmonar en las ratas expuestas en el año 2009 fue menor que el observado en el año $2005^{2}$, lo cual podría explicarse por una diferente composición de los contaminantes especialmente del material particulado, ya que las concentraciones promedio por hora calculadas para $\mathrm{PM}_{10}$ en el sitio de exposición fueron similares $73,1 \mu \mathrm{g} / \mathrm{m}^{3}$ en 2005 versus 83,1 en 2009.

En el presente estudio es interesante destacar que aunque no hubo daño pulmonar histológicamente detectable, al cabo de 90 días de exposición se encontró un aumento pequeño, pero significativo de la viscosidad y de la proteína C reactiva plasmáticas y del hematocrito. El aumento de proteína $\mathrm{C}$ reactiva (Tabla 1) podría indicar el inicio de un estado proinflamatorio que si estuviese asociado a aumento de fibrinógeno, podría explicar el discreto incremento de la viscosidad plasmática. Por otra parte, el aumento del microhematocrito podría también estar relacionado con el estado proinflamatorio de las ratas expuestas que al aumentar su metabolismo, podría haber inducido aumento de la ventilación minuto y de la pérdida insensible de agua que eventualmente provocaría una discreta disminución del volumen plasmático que se reflejaría en el hematocrito.

El aumento de material particulado se ha asociado a un estado proinflamatorio y procoagulante en estudios previos realizados en seres humanos ${ }^{12}$. En el presente estudio la concentración de $\mathrm{PM}_{10}$ sobrepasó sistemáticamente la norma nacional anual y frecuentemente la norma diaria, en tanto que la concentración de $\mathrm{PM}_{2,5}$ superó habitualmente la norma anual propuesta por la CONAMA. Sólo en uno de los 90 días de exposición el $\mathrm{NO}_{2}$ superó su norma anual, en tanto que el $\mathrm{CO}$ y el ozono siempre estuvieron por debajo de la norma de 8 horas de exposición. Por lo tanto, el efecto proinflamatorio observado parecería estar relacionado fundamentalmente con la contaminación por material particulado.

Por otra parte, en pacientes con cardiopatía coronaria, se ha detectado que el aumento de $\mathrm{PM}_{2,5}$ del aire de Santiago promueve la inflamación (aumento de proteína $\mathrm{C}$ reactiva), la hemoconcentración (aumento del hematocrito) y estimula mecanismos trombogénicos (aumento de las plaquetas y viscosidad); en sujetos sin cardiopatía coronaria sólo se constató aumento de las plaquetas y de la viscosidad plasmática en tanto que la proteína $\mathrm{C}$ reactiva disminuyó ${ }^{13}$. Es interesante destacar que algunas de las alteraciones encontradas en santiaguinos con patología coronaria ${ }^{13}$ son similares a las encontradas en las ratas de la serie expuesta del presente estudio, llamando la atención que en los sujetos sin patología coronaria sólo el aumento de la viscosidad plasmática sea coincidente con los resultados obtenidos en las ratas expuestas, en las cuales observamos además aumento de la proteína $\mathrm{C}$ reactiva y del microhematocrito. Lo cual podría indicar una eventual mayor sensibilidad del modelo experimental de ratas centinela que el ser humano a los efectos sistémicos de la contaminación aérea de Santiago. Sin embargo, debemos ser cautos en la eventual extrapolación de los efectos de los contaminantes observados en la rata a otras especies incluidos los seres humanos. En efecto, sin entrar a considerar otras diferencias fisiológicas y bioquímicas, la rata tiene un consumo de $\mathrm{O}_{2}$ por unidad de masa corporal 20,2 veces mayor que el del ser humano (76 versus $3,75 \mathrm{~mL}$ de $\mathrm{O}_{2} \cdot \mathrm{kg}^{-1} \cdot \mathrm{min}^{-1}$ ) su frecuencia respiratoria es siete veces más elevada que la de una persona adulta normal ( 85 versus 12 ciclos . $\left.\mathrm{min}^{-1}\right)^{14}$. Por lo tanto, es necesario hacer ajustes interespecies cuando se intenta extrapolar al ser humano resultados sobre evaluación de riesgo obtenidos en mamíferos pequeños ${ }^{15}$.

Otro factor a considerar en el análisis de los resultados obtenidos es la eventual participación de factores confundentes durante la exposición 
intermitente de ratas de laboratorio al aire urbano de una avenida de Santiago con elevado tráfico vehicular (1.700 vehículos/hora). De estos factores el más relevante es el nivel de ruido ambiental, mucho más alto que el de su habitat del bioterio, lo cual constituiría un factor adicional de estrés para estos animales.

Se ha postulado ${ }^{16}$ que la exposición a partículas ultrafinas $(<0,1 \mu \mathrm{m})$ derivadas de combustibles fósiles habitualmente usados por los vehículos motorizados, pueden inducir una inflamación sistémica a través de estrés oxidativo en respuesta a la producción de especies reactivas del oxígeno, derivadas de los componentes de óxido-reducción (cobre, níquel, hierro, vanadio y quinonas entre otros) de estas partículas que por su tamaño pueden alcanzar fácilmente el lecho vascular de diferentes órganos, además del pulmón.

En nuestros experimentos determinamos la concentración plasmática de $\mathrm{F}_{2}$-isoprostano, que ha sido comúnmente utilizado como un indicador de estrés oxidativo y de daño pulmonar agudo ${ }^{11}$, encontrándose aumentado en sujetos normales expuestos a ozono, a alergenos y a humo de cigarrillo $^{17}$. Considerando estos antecedentes esperábamos encontrar un aumento de $\mathrm{F}_{2}$-isoprostano en las ratas expuestas. Sin embargo, la concentración plasmática de $\mathrm{F}_{2}$-isoprostano no se modificó significativamente al cabo de 90 días de exposición intermitente al ser comparadas con los controles. Una posible explicación para esta ausencia de respuesta podría ser una adaptación al estrés oxidativo, provocada por la exposición intermitente a los contaminantes aéreos, hecho que se ha descrito en la atenuación de la respuesta ante la exposición repetida a ozono y que se denomina "preacondicionamiento al estrés oxidativo por ozono", en la generación de este fenómeno el aumento de la producción de antioxidantes y la proliferación de células alveolares tipo II podrían jugar un papel importante ${ }^{18}$.

Este estudio sugiere que la exposición intermitente de ratas centinela a los contaminantes del tráfico vehicular al cabo de un período de 90 días provoca aumento significativo -en comparación con ratas no expuestas- de marcadores proinflamatorios tales como la proteína $\mathrm{C}$ reactiva $\mathrm{y}$ viscosidad plasmática, que aunque no alcanzaron a comprometer el desarrollo de la curva de peso corporal de los animales expuestos, serían indicadores de un daño inflamatorio sistémico inicial, el cual podría preceder a la disminución del crecimiento corporal y al daño histopatológico pulmonar detectado en otro de nuestros estudios en esta misma especie con la exposición a contaminantes del tráfico vehicular urbano.

\section{Agradecimientos}

Agradecemos a los Srs. Rodrigo Seguel y Sergio Ibarra, Centro Nacional del Medio Ambiente (CENMA), Universidad de Chile, el habernos aportado la información sobre contaminación atmosférica registrada en las estaciones de la red de monitoreo de la Región Metropolitana. También agradecemos la participación en este estudio del Sr. Jorge Guzmán O., ayudante técnico de nuestro laboratorio y de los Srs. Nicolás Pinochet G., Andrés Sanhueza Z. y Yoshiro Sato A., alumnos del Curso Unidades de Investigación 2009, Escuela de Medicina, Universidad de Chile.

\section{Bibliografía}

1.- MORALES RG. Introducción a la contaminación atmosférica urbana: ciudad de Santiago. En: Contaminación atmosférica urbana. Episodios críticos de contaminación ambiental en la ciudad de Santiago. Morales RG Ed. Editorial Universitaria, Santiago de Chile, 2006; pp 17-34.

2.- OYARZÚN M, DUSSAUBAT N, MILLER M E, ULRIKSEN P, GONZÁLEZ S. Lung damage in rats exposed to urban air pollution in Santiago de Chile. Eur Respir J 2006; (Suppl 50): 619S.

3.- POPE C A, BURNETT R T, THURSTON G D, THUN M J, CALLE E E, KREWSKI D, et al. Cardiovascular mortality and long-term exposure to particulate airpollution: epidemiological evidence of general pathophysiological pathways of disease. Circulation 2004; 109: 71-7.

4.- PETERS A, DORING A, WICHMANN H E, KOENIG W. Increased plasma viscosity during air pollution episode: a link to mortality? Lancet 1997; 349: 1582-7.

5.- PETERS A, FROHLICH M, DORING A, IMMERVOLL T, WICHMANN H E, HUTCHINSON W L, et al. Particulate air pollution is associated with an acute phase response in men: results from the MONICA Augsburg study. Eur Heart J 2001; 22: 1198-204.

6.- VAN DER SCHALIE $W \mathrm{H}$, GARDNER H S Jr, BANTLE JA, DE ROSA C T, FINCH RA, REIF J S, et al. Animals as sentinels of human health hazards of environmental chemicals. Environ Health Perspect 1999; 107: 309-15.

7.- MORROW J D, FREI B, LONGMIRE A W, GAZIANO J M. LYNCH S M, SHYR Y. Increase in circulating products of lipid peroxydation (F2-isoprostane) in smokers. N Engl J Med 1995; 332: 1198-203.

8.- DOWTON S B, COLTEN H R. Acute phase reactants in inflammation and infection. Seminars in Hematology1988; 25: 84-90.

9.- MATUS P, LUCERO R. Normas primarias de calidad del aire. Rev Chil Enf Respir 2002; 18: 112-22.

10.- SAPAG A. Aprueba anteproyecto de norma primaria de calidad ambiental para material particulado respirable 
$\mathrm{MP}_{2,5}$. Resolución exenta $\mathrm{N}^{\circ} 4624$ CONAMA. 10 de agosto, 2009.

11.- JANSSEN L J. Isoprostanes and lung vascular pathology. Am J Respir Cell Mol Biol 2008; 39: 383-9.

12.- HOFFMANN B, MOEBUS S, DRAGANO N, STANG A, MÖHLENKAMP S, SCHMERMUND A, et al. Chronic residential exposure to particulate matter air pollution and systemic inflammatory markers. Environ Health Perspect 2009; 117: 1302-8.

13.- JALIL J, DUMASA E, CIFUENTES L, OCARANZA MP, CHAMORRO G, PADILLA I, et al. Desbalance autonómico y procoagulación debido a contaminación atmosférica en Santiago: un estudio prospectivo. Rev Chil Cardiol 2003; 22: 223-32.

14.- ALTMAN P L, DITTMER D S. Respiration and Circulation. Biological Handbooks. Bethesda, Maryland, USA. 1971; págs: 53-59 y 327-332.
15.- PIPELKO W E. Feasibility of dose adjustment based on differences in long term clearance rates of inhaled particulate matter in humans and laboratory animals. Regul Toxicol Pharmacol 1987; 7: 236-52.

16.- DELFINO R J, SIOUTAS C, MALIK S. Potential role of ultrafine particles in associations between airborne particle mass and cardiovascular health. Environ Health Perspect 2005; 113: 934-46.

17.- PILZ H, OGUOGHO A, CHEHNE F, LUPATTELLI G, PALUMBO B, SINZINGER H. Quitting cigarette smoking results in a fast improvement of in vivo oxidation injury (determined via plasma, serum and urinary isoprostane). Thrombosis Research 2000; 99: 209-21.

18.- WIESTER M, TEPPER J, DOERFLER D, COSTA D. Ozone adaptation in rat after chronic exposure to a simulated urban profile of ozone. Fundam Appl Toxicol 1995; 34: 42-51.

Correspondencia a:

Dr. Manuel Oyarzún G.

Programa de Fisiopatología, ICBM,

Facultad de Medicina, Universidad de Chile.

Avda. Salvador 486. Providencia, Santiago.

E-mail: moyarzun@med.uchile.cl 